\title{
EMPLEO DEL PREPARADO 114 T.E. COMO ABREVIADOR DEL PARTO
}

\author{
Dr. Jesús Alberto Gómez Palacino* \\ Dr. Fernando Sánchez Torres*
}

El objeto del presente estudio es presentar los resultados obtenidos por la investigación clínica del empleo de un nuevo espasmolítico de sintesis como abreviador del parto: el 114 T.E. O Yoduro de Alfa-tienil-1fenil-1.N metil morfilinium 3 propanol $1(x)$.

El 114 T.E. es un componente de los Ilamados "espasmolíticos"; anticolinérgico con un poder antiespasmódico mayor al de la papaverina y parasimpaticolítico superior al de la atropina y al N. Butil bromuro de hioscina.

Su efecto se ejerce por medio de 4 mecanismos:

1?. Acción directa sobre la fibra muscular lisa.

2: Acción sobre el mediador químico.

3: Acción sobre la fibra post-ganglionar.

4: Acción directa sobre los plexos intestinales.

\section{Material}

El trabajo se realizó en el Instituto Materno Infantil, "Concepción Villaveces de Acosta", en Bogotá.

Se estudió su efecto en un total de 100 pacientes seleccionadas en dos grupos así:

GRUPO A - Cincuenta (50) primigestantes con embarazo a término, con feto vivo en presentación de vértice, membranas íntegras, en franco trabajo de parto y sin ningún problema de orden obstétrico o general.

GRUPO B - Cincuenta (50) multigestantes (tercero o cuarto embarazo) que cumplieron los mismos requisitos del grupo $A$.

\section{Método}

Se utilizó el preparado comercial 114 T.E., que contiene 5 mgr. de sustancia activa por ampolla, en inyección intravenosa y dosis única.

El estudio se hizo en forma ciega, es decir, 25 pacientes del grupo A y 25 del grupo B recibieron el 114 T.E., y otro número igual de cada grupo recibió un placebo. Los investigado-

* Del Departamento de Obstetricia y Ginecología de la Facultad de Medicina (U. N.).

(x) "Visceralgina" de "Laboratorios Hormona" S. A. 
res ignoraron cuales ampollas correspondían al 114 T.E. y cuales al placebo. A las pacientes del grupo A se les aplicó el contenido de una ampolla del 114 T.E. 0 del placebo cuando el cuello uterino estuvo borrado y hubo alcanzado una dilatación de 4 a $5 \mathrm{cms}$., previa amniotomía artificial y previa comprobación clínica de las características de la contracción uterina. Posteriormente el registro gráfico de las contracciones y el tacto vaginal practicado a intervalos aproximados de media a una hora permitieron controlar la evolución del parto.

El mismo método se siguió con las pacientes del grupo $B$, no siendo re- quisito indispensable en ellas que el cuello uterino estuviese borrado completamente antes de aplicar el 114 T.E. O el placebo.

Los datos necesarios, de acuerdo con la metodología, se anotaron en la historia clínica e incluyeron: hora de amniotomía, hora exacta de aplicación de la droga o del placebo, la hora en que se completó la dilatación cervical y la hora del nacimiento. Quedó consignada la variedad de posición, el peso y el sexo fetales y la calificación de Apgar. Se registraron además las manifestaciones consecutivas a la administración de la droga.

\section{Casuística}

TABLA 1

PACIENTES PRIMIgESTANTES QUE RECIBIERON 114 T.E.

\begin{tabular}{|c|c|c|c|c|c|c|c|}
\hline \multirow[b]{2}{*}{ Caso } & \multirow[b]{2}{*}{$\begin{array}{l}\text { Variedad de } \\
\text { posición }\end{array}$} & \multirow[b]{2}{*}{$\begin{array}{l}\text { Duración hasta } \\
\text { Dilatación completa }\end{array}$} & \multirow{2}{*}{$\begin{array}{l}\text { Duración } \\
\text { del período } \\
\text { expulsivo }\end{array}$} & Duración & \multirow[b]{2}{*}{ Peso } & \multirow[b]{2}{*}{ Apgar } & \multirow[b]{2}{*}{$\begin{array}{c}\text { Efectos } \\
\text { secundarios }\end{array}$} \\
\hline & & & & $\begin{array}{l}\text { total del } T \text {. } \\
\text { de parto }\end{array}$ & & & \\
\hline 1 & ODA & $55^{\prime}$ & $40^{\prime}$ & $8 \mathrm{H} .10^{\prime}$ & 3.000 & 10 & \\
\hline 2 & ODP & $120^{\prime}$ & $40^{\prime}$ & $6 \mathrm{H}^{\prime} .10^{\prime}$ & 2.500 & 10 & \\
\hline 3 & ODP & $80^{\prime}$ & $60^{\prime}$ & $5 \mathrm{H} .50^{\prime}$ & 2.500 & 10 & \\
\hline 4 & ODA & 20 & $20^{\prime}$ & $4 \mathrm{H}$. & 2.800 & 10 & \\
\hline 5 & ODA & $15^{\prime}$ & $40^{\prime}$ & $8 \mathrm{H}$. & 3.000 & 10 & \\
\hline 6 & OIA & $65^{\prime}$ & $80^{\prime}$ & $10 \mathrm{H} .35^{\prime}$ & 3.400 & 8 & \\
\hline 7 & OIT & $55^{\prime}$ & $50^{\prime}$ & $9 \mathrm{H}$. & 3.200 & 9 & \\
\hline 8 & OIA & $30^{\prime}$ & $20^{\prime}$ & $5 \mathrm{H} .50^{\prime}$ & 2.000 & 10 & \\
\hline 9 & ODP & $25^{\prime}$ & $50^{\prime}$ & 9 H. $55^{\prime}$ & 2.900 & 10 & \\
\hline 10 & OIA & $105^{\prime}$ & $25^{\prime}$ & $7 \mathrm{H}$. & 2.700 & 10 & \\
\hline 11 & OIA & $25^{\prime}$ & $30^{\prime}$ & $8 \mathrm{H}$. & 2.600 & 10 & \\
\hline 12 & ODP & $15^{\prime}$ & $5^{\prime}$ & $5 \mathrm{H} .30^{\prime}$ & 2.800 & 10 & \\
\hline 13 & ODP & $20^{\prime}$ & $15^{\prime}$ & $6 \mathrm{H}$. & 3.050 & 10 & \\
\hline 14 & OIT & $90^{\prime}$ & $30^{\prime}$ & $13 \mathrm{H}$. & 2.700 & 10 & \\
\hline 15 & ODP & $70^{\prime}$ & $25^{\prime}$ & $7 \mathrm{H}$. & 2.800 & 10 & \\
\hline 16 & OIA & $40^{\prime}$ & $10^{\prime}$ & $8 \mathrm{H}$. & 2.900 & 9 & Náuseas \\
\hline 17 & ODA & $135^{\prime}$ & $30^{\prime}$ & 7H. $20^{\prime}$ & 3.050 & 10 & \\
\hline 18 & OIA & $35^{\prime}$ & $5^{\prime}$ & $8 \mathrm{H} .45^{\prime}$ & 3.445 & 10 & \\
\hline 19 & ODA & $55^{\prime}$ & $15^{\prime}$ & 5 H. $40^{\prime}$ & 3.600 & 10 & \\
\hline 20 & OIT & $45^{\prime}$ & $65^{\prime}$ & 7H. 50' & 2.800 & 10 & \\
\hline 21 & OID & $140^{\prime}$ & $55^{\prime}$ & 7 H. $20^{\prime}$ & 3.100 & 7 & \\
\hline 22 & OIT & $80^{\prime}$ & $65^{\prime}$ & 개. $45^{\prime}$ & 2.640 & 8 & \\
\hline 23 & OIA & $85^{\prime}$ & $80^{\prime}$ & $8 \mathrm{H} .5^{\prime}$ & 2.840 & 10 & \\
\hline 24 & ODT & $110^{\prime}$ & $70^{\prime}$ & $7 \mathrm{H}^{\prime} .10^{\prime}$ & 3.100 & 10 & \\
\hline 25 & ODP & $150^{\prime}$ & $60^{\prime}$ & 7H. $30^{\prime}$ & 3.000 & 7 & \\
\hline
\end{tabular}


TABLA 2

PACIENTES PRIMIGESTANTES QUE RECIBIERON PLACEBO

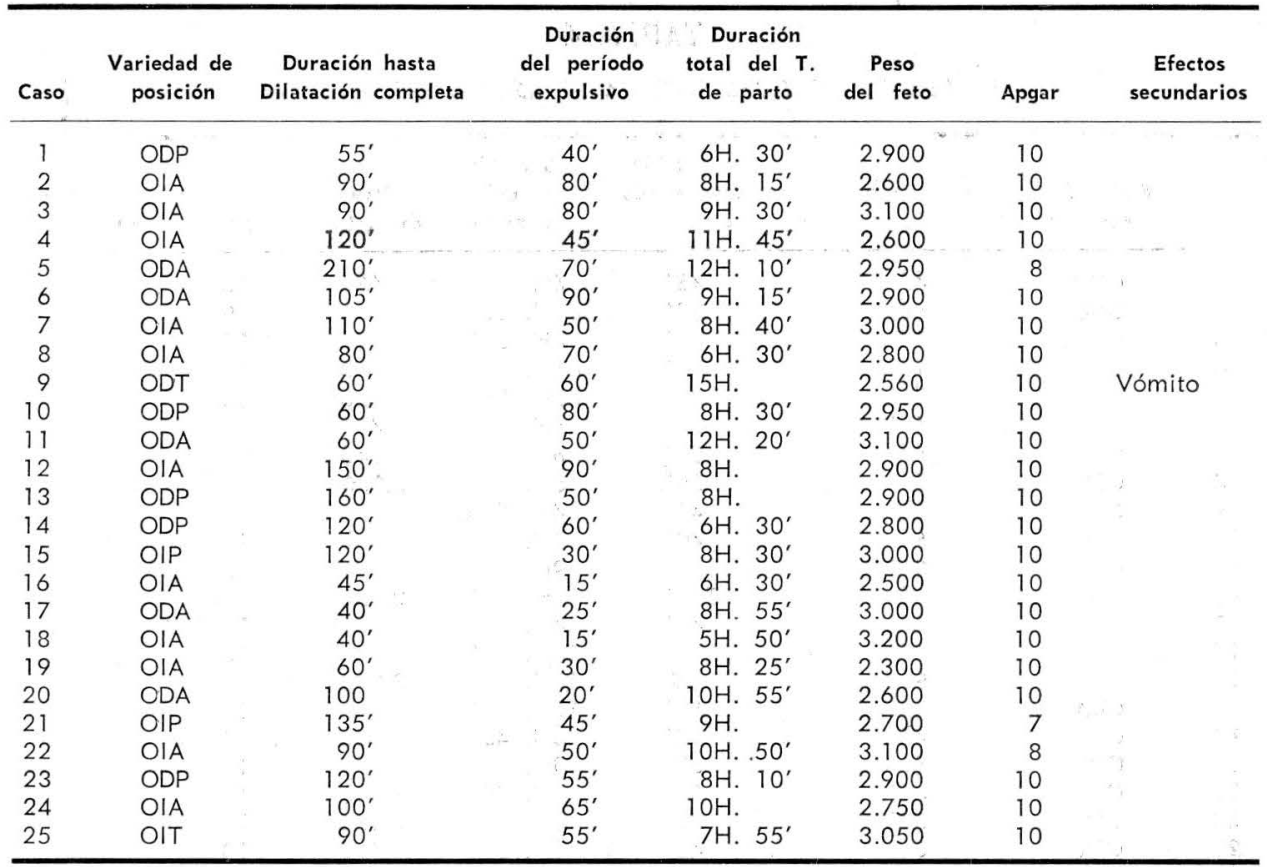

TABLA 3

PACIENTES MULTIGESTANTES QUE RECIBIERON 114 T.E.

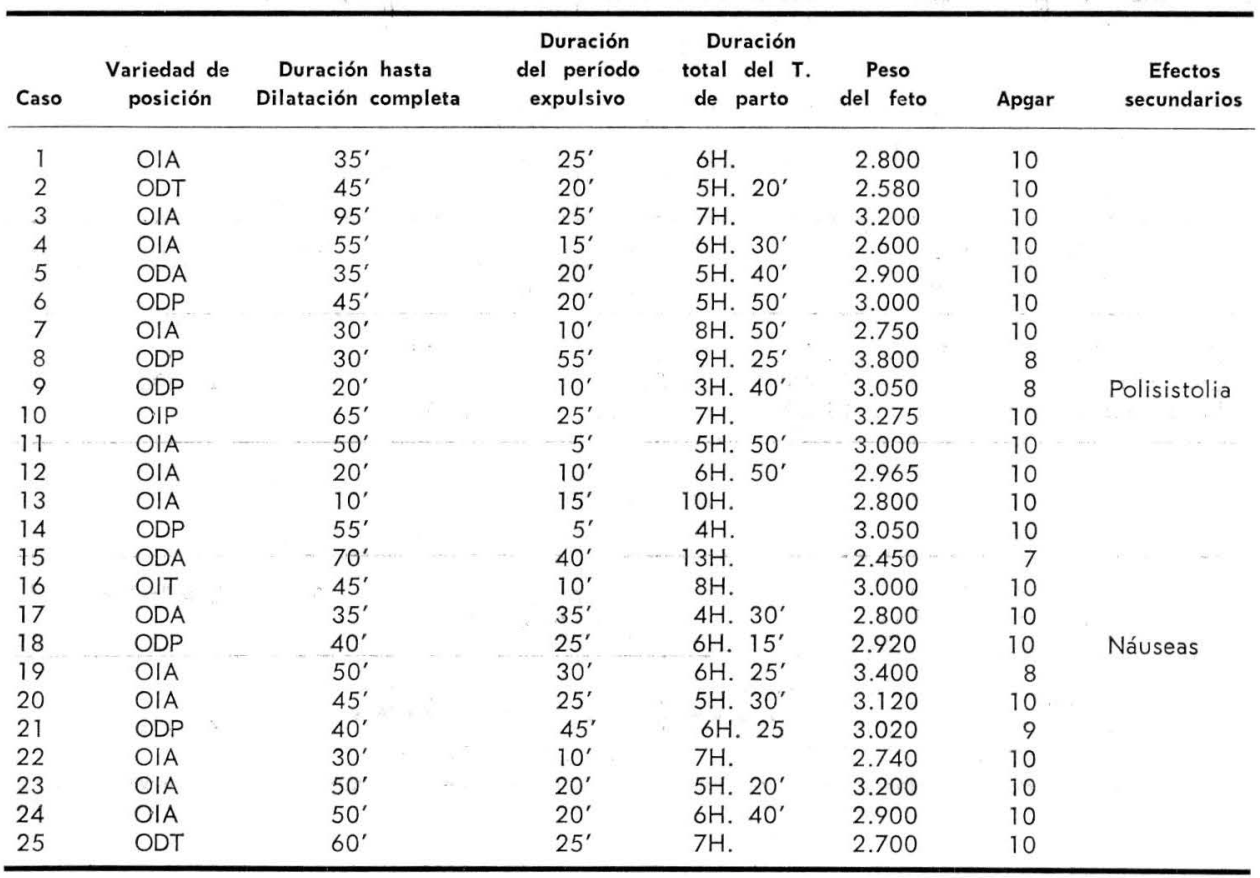


TABLA 4

PACIENTES MULTIGESTANTES QUE RECIBIERON PLACEBO

\begin{tabular}{|c|c|c|c|c|c|c|c|}
\hline Caso & $\begin{array}{l}\text { Variedad de } \\
\text { posición }\end{array}$ & $\begin{array}{l}\text { Duración hasta } \\
\text { Dilatación completa }\end{array}$ & $\begin{array}{l}\text { Duración } \\
\text { del período } \\
\text { expulsivo }\end{array}$ & $\begin{array}{l}\text { Duración } \\
\text { total del } T \text {. } \\
\text { de parto }\end{array}$ & $\begin{array}{c}\text { Peso } \\
\text { del feto }\end{array}$ & Apgar & $\begin{array}{c}\text { Efectos } \\
\text { secundarios }\end{array}$ \\
\hline 1 & ODP & $45^{\prime}$ & $45^{\prime}$ & $6 \mathrm{H} .10^{\prime}$ & 2.680 & 10 & \\
\hline 2 & OIA & $60^{\prime}$ & $30^{\prime}$ & $5 \mathrm{H} .10^{\prime}$ & 3.600 & 9 & \\
\hline 3 & OIA & $95^{\prime}$ & $40^{\prime}$ & $4 \mathrm{H} .30^{\prime}$ & 3.400 & 10 & \\
\hline 4 & ODP & $10^{\prime}$ & $50^{\prime}$ & $6 \mathrm{H}$. & 2.900 & 10 & \\
\hline 5 & OIP & $70^{\prime}$ & $45^{\prime}$ & 개. $25^{\prime}$ & 3.480 & 10 & \\
\hline 6 & ODP & $60^{\prime}$ & $60^{\prime}$ & $5 \mathrm{H}$ & 2.480 & 10 & \\
\hline 7 & OIA & $60^{\prime}$ & $35^{\prime}$ & $6 \mathrm{H}$. & 2.640 & 10 & \\
\hline 8 & OIA & $30^{\prime}$ & $25^{\prime}$ & $6 \mathrm{H}$. & 2.900 & 10 & Náuseas \\
\hline 9 & OIP & $100^{\prime}$ & $40^{\prime}$ & $4 \mathrm{H} .50^{\prime}$ & 2.980 & 10 & \\
\hline 10 & OIA & $55^{\prime}$ & $40^{\prime}$ & $7 \mathrm{H}$. & 2.800 & 10 & $\therefore$ \\
\hline 11 & OIP & $30^{\prime}$ & $110^{\prime}$ & $9 \mathrm{H}$. & 3.300 & 8 & \\
\hline 12 & ODT & $135^{\prime}$ & $100^{\prime}$ & $14 \mathrm{H} .55^{\prime}$ & 2.750 & 8 & \\
\hline 13 & ODT & $240^{\prime}$ & $45^{\prime}$ & $14 \mathrm{H}$ & 3.500 & 10 & \\
\hline 14 & OIP & $60^{\prime}$ & $60^{\prime}$ & $10 \mathrm{H} .15^{\prime}$ & 2.640 & 10 & \\
\hline 15 & OIA & $90^{\prime}$ & 65 & $13 \mathrm{H}$. & 3.000 & 10 & \\
\hline 16 & ODA & $60^{\prime}$ & $50^{\prime}$ & $12 \mathrm{H} .50^{\prime}$ & 2.800 & 10 & \\
\hline 17 & OIA & $45^{\prime}$ & $15^{\prime}$ & $8 \mathrm{H} .45^{\prime}$ & 3.050 & 7 & \\
\hline 18 & ODT & $60^{\prime}$ & $60^{\prime}$ & $10 \mathrm{H}$ & 2.650 & 10 & \\
\hline 19 & OIP & $30^{\prime}$ & $55^{\prime}$ & 9H. 55' & 3.850 & 10 & \\
\hline 20 & OIA & $50^{\prime}$ & $10^{\prime}$ & $3 \mathrm{H}$. & 2.680 & 10 & \\
\hline 21 & OIA & $30^{\prime}$ & $45^{\prime}$ & $6 \mathrm{H} .15^{\prime}$ & 3.300 & 10 & \\
\hline 22 & OIP & $30^{\prime}$ & $80^{\prime}$ & $4 \mathrm{H} .50^{\prime}$ & 2.900 & 8 & \\
\hline 23 & OIA & $40^{\prime}$ & $15^{\prime}$ & 3 H. $55^{\prime}$ & 2.700 & 10 & \\
\hline 24 & ODT & $120^{\prime}$ & $30^{\prime}$ & 4 H. $50^{\prime}$ & 3.500 & 10 & \\
\hline 25 & OIA & $10^{\prime}$ & $5^{\prime}$ & $5 \mathrm{H} .30^{\prime}$ & 2.500 & 10 & \\
\hline
\end{tabular}

TABLA 5 - RESULTADOS

PRIMIGESTANTES

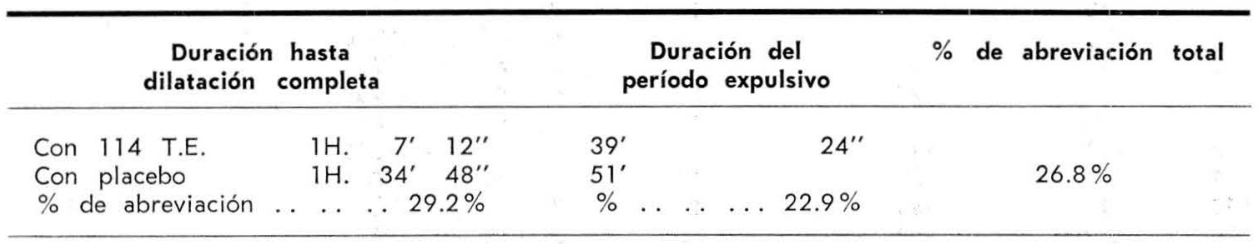

MULTIGESTANTES

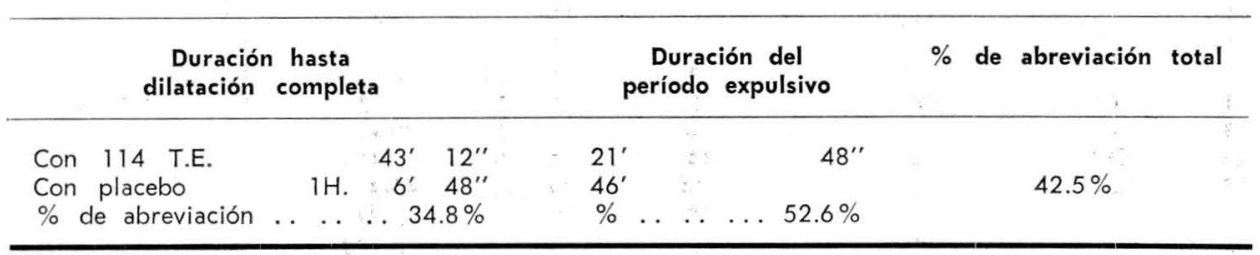


Con el fin de hacer más objetivos los efectos del medicamento se registró gráficamente la actividad uterina por medio del tocógrafo de Lorand y para hacer más depurada la investigación las pacientes no recibieron ninguna otra medicación sedante ni analgésica. Se descartaron aquellos casos que terminaron de manera intervenida.

\section{Comentario y Conclusiones}

El número limitado de casos, 25 para cada grupo de pacientes, no permite obtener la prueba concluyente de significación estadística en cuanto a la abreviación de los distintos tiempos, sinembargo, el compuesto i 14 T.E. acortó los tiempos de dilatación y expulsión y por consiguiente la duración total del trabajo del parto.

Si como antes se dijo, el período de expulsión fue más corto en las pacientes que recibieron 114 T.E. que el de aquellas a quienes se administró placebo, se puede concluir que su acción se ejerció no solamente sobre la dilatación cervical sino también sobre la dinámica del cuerpo uterino, tal como pudo observarse en algunos registros de la contracción.

Sus efectos fueron igualmente observados tanto en pacientes primigestantes como en multigestantes.

La calificación del recién nacido por el método de Apgar (color, tono, llanto, frecuencia respiratoria y cardíaca en el primer minuto de vida), permite concluir que el medicamento no tuvo ningún efecto nocivo sobre el producto porque las cifras inferiores a 10 fueron igualmente observadas en el grupo en experimentación como en el grupo control.

Por último, no se apreciaron en las pacientes efectos secundarios con el empleo del medicamento.

\section{RESUMEN}

Se presentan los resultados obtenidos en 100 casos obstétricos correspondientes a pacientes en trabajo de parto, la mitad de las cuales recibieron la droga en experimentación clínica (114 T.E.) y la otra mitad recibió placebos. La vía de administración fué siempre la venosa en dosis de $5 \mathrm{mgr}$. y las pacientes, en igualdad de condiciones, eran normales tanto desde el punto de vista general como en su aspecto obstétrico. Cincuenta de ellas eran primigestantes y cincuenta multigestantes.

Se registró la actividad uterina por medio del tocógrafo de Loránd y se utilizaron como parámetros comparativos los tiempos de dilatación cervical a partir de $5 \mathrm{~cm}$. y la duración del período expulsivo; se obtuvo el porcentaje de abreviación.

Se observaron además los posibles efectos secundarios sobre la madre y la acción de la medicación sobre el recién nacido. 